\title{
Progress in studies of intergalactic He II absorption
}

\author{
Wei Zheng ${ }^{1}$, S. F. Anderson ${ }^{2}$, G. A. Kriss ${ }^{3}$, K. Chiu ${ }^{1}$, \\ D. Haggard ${ }^{2}$, D. P. Schneider ${ }^{4}$, C. J. Hogan ${ }^{2}$, D. G. York ${ }^{5}$, \\ S. Burles ${ }^{6}$, and A. Meiksin ${ }^{7}$ \\ ${ }^{1}$ Department of Physics and Astronomy, Johns Hopkins University, \\ Baltimore, MD 21218, USA \\ email: zheng@pha.jhu.edu \\ ${ }^{2}$ Department of Astronomy, University of Washington, Seattle, WA 98195, USA \\ ${ }^{3}$ Space Telescope Science Institute, Baltimore, MD 21218, USA \\ ${ }^{4}$ Department of Astronomy and Astrophysics, Pennsylvania State University, \\ University Park, PA 16802, USA \\ ${ }^{5}$ Department of Astronomy, University of Chicago, Chicago, IL 60637, USA \\ ${ }^{6}$ Department of Physics, Massachusetts Institute of Technology, Cambridge, MA 02139, USA \\ ${ }^{7}$ Institute for Astronomy, University of Edinburgh, Blackford Hill, Edinburgh EH9 3HJ, UK

\begin{abstract}
Significant progress has been made in the last decade toward an understanding of the intergalactic He II absorption. At redshifts $z<2.8$, individual absorbers are found to be ionised mainly by a hard quasar continuum, but their ionisation states vary considerably and the ionising radiation field may have contributions from a soft stellar continuum. The He II opacity increases significantly at $z \sim 2.8$, suggesting a possible end of the general reionisation of intergalactic He II. Using the massive SDSS database and UV selection with HST and GALEX, we have found new quasars at $z>3.5$, with detected He II absorption. Future observations will pinpoint the epoch of reionisation of the intergalactic He II.
\end{abstract}

\section{Introduction}

Helium is the second most abundant element in the Universe, and He II absorption is the most sensitive tracer of the intergalactic medium. At redshifts $z>2$, the He II feature in quasar spectra is observable in the far-UV band. However, the search for He II absorption in quasars is exceptionally challenging: the lines of sight toward the majority of quasars at $z>2$ are not transparent at the wavelength of He II Ly $\alpha$. The UV flux of any high- $z$ object is inevitably attenuated by accumulated absorption of numerous H I Ly $\alpha$ forest lines. In addition, H I absorption from intervening Lyman-limit systems (LLS) may severely cut off the flux from any wavelengths short-ward of the quasar's Ly $\alpha$ emission. The identification of the rare quasars having the truly clean lines of sight needed for He II studies requires large sky surveys and followup UV screening.

The first presence of a Gunn-Peterson trough was detected in He II absorption, in a quasar at $z=3.28$ (Jakobsen et al. 1994). Over the last decade eight quasars have been found with He II absorption (Table 1). The observations of these quasars with HST, FUSE, and other UV instruments have accumulated more than one million seconds and provided invaluable insight into the IGM properties at $z \sim 3$. 
Table 1. Quasars with HeII absorption

\begin{tabular}{l|c|c|}
\hline Object & Redshift & Observation \\
\hline HS1700+64 & 2.74 & HUT,FOS,HRS,STIS,FUSE \\
HE 2347-43 & 2.88 & HRS,STIS,FOS,FUSE \\
CSO 0118 & 2.98 & STIS \\
PKS 1935-69 & 3.18 & HRS,STIS,FOS,FOC \\
Q 0302-00 & 3.29 & HRS,STIS,FOS,FOC \\
SDSS 2346-00 & 3.51 & STIS,ACS* \\
SDSS 1711+60 & 3.82 & STIS,ACS* \\
SDSS 1614+48 & 3.82 & STIS \\
\hline
\end{tabular}

* To be observed soon

\section{The ionising radiation field at $z<3$}

The FUSE spectrum of quasar HS2347-43 (Kriss et al. 2001; Zheng et al. 2004) enables us to identify hundreds of absorption features between $2.2<z<2.7$. The He II to $\mathrm{H}$ I column density ratio ranges from 1 to $>1000$, with an average of $\sim 55$. Ratios of $<100$ are consistent with photoionisation of the absorbing gas by a hard ionising spectrum resulting from the integrated light of quasars, but ratios of $>100$ in many locations indicate additional contributions from starburst galaxies or heavily filtered quasar radiation. By a redshift of 2 , the ionisation of intergalactic $\mathrm{He}^{+}$is exclusively due to quasars.

At redshifts $z>2.8$, the He II optical depth shows a sudden increase, in a manner similar to that observed at $z \sim 6$ for H I (Fan et al. 2002). The He II Ly $\alpha$ optical depths in the opaque regions of the UV spectrum at $z>2.7$ is measured to have values of $\tau_{\text {Ly } \alpha} \sim 5$ using the corresponding troughs in the Ly $\beta$ portions of the spectrum to extend our dynamic range. This sudden but finite increase in optical depth suggests that we are seeing the end of the He II reionisation process that began at an earlier epoch as indicated indirectly by the increase in $\mathrm{H}$ I line widths and $\mathrm{H}$ I opacity observed at $z=3.2-3.4$ (Theuns et al. 2002).

\section{The SDSS/HST/GALEX search at $z>3$}

The Sloan Digital Sky Survey (SDSS) has produced the largest quasar catalogue to date (Schneider et al. 2003), and the number quasars is growing every month. It provides an excellent sample in which to search for quasar candidates with He II absorption features. We have carried out a snapshot search using the STIS instrument aboard HST. About 80 quasars have been screened in the UV. We have found three quasars with detected UV flux near the wavelength of redshifted Ly $\alpha$ in their snapshot HST spectra (see Table 1 and Fig. 1). We plan longer exposures using the ACS prism to study the details of He II absorption at redshifts as high as 3.8 .

The UV imaging data of GALEX now provide an additional avenue to check the UV detectability of SDSS quasars. Based on the GALEX GR1 database and SDSS DR3, we have independently confirmed more than a dozen quasars with detected near-UV flux. It is anticipated that the SDSS-based searches will yield 50-100 quasars at $z>3$ that are suitable for UV followup. The lines of sight towards these quasars will provide new insight into the reionisation status of intergalactic $\mathrm{He}$ II. If $\mathrm{He}^{+}$is not fully ionised at these redshifts, damped absorption profiles are expected, which can be seen even at low spectral resolution. We anticipate that the epoch of reionisation of intergalactic He II may be observationally determined in the next decade. 


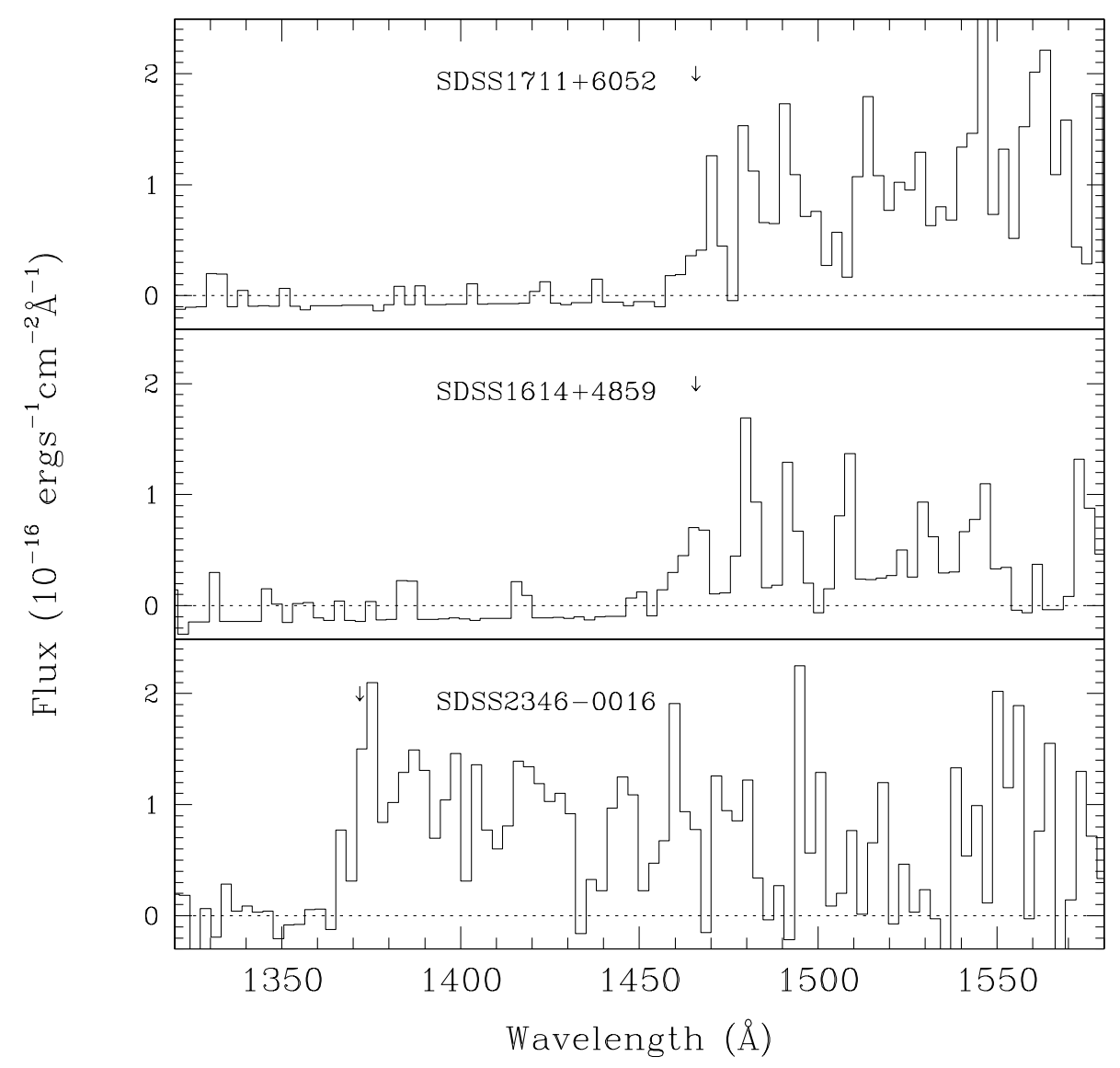

Figure 1. Snapshot (10 minute) STIS spectra of three quasars. The G140L data are binned by nine pixels. The He II Ly $\alpha$ absorption edge in each panel is marked by an arrow. These new targets were discovered by a HST/SNAP search project, and they represent the highest known redshifts for He II absorption.

\section{Acknowledgements}

Support for this research was provided by NASA through grants 8582, 9067, 9759, and 10132 from the Space Telescope Science Institute, which is operated by the Association of Universities for Research in Astronomy, Inc., under NASA contract NAS 5-26555. Support was also provided through NASA/GALEX grant NNG05GE12G.

\section{References}

Fan, X., et al., 2002, AJ, 125, 1649

Jakobsen, P., et al., 1994, Nature, 370, 35

Kriss, G. A., et al., 2001, Science, 293, 1112

Reimers, D., et al., 1997, A\&A, 327, 890

Schneider, D. P., et al., 2003, AJ, 126, 2579

Theuns, T., et al., 2002, ApJ, 567, 103

Zheng, W., et al., 2004, ApJ, 605, 631 